\title{
Influential Article Review - Personal and Household Abuse Prevention
}

\author{
Tommy Cooke
}

\author{
Jaylan Bannister
}

Hawwa Miller

This paper examines domestic violence. We present insights from a highly influential paper. Here are the highlights from this paper: How police respond to domestic and family violence (DFV) has important ramifications for the safety of women and their children. Despite recognition of the key role police play in responding to DFV and the importance of the perspectives of survivors in informing appropriate responses, there has been no recent exploration of survivors' experiences of the police response to DFV in Queensland. This article draws on interviews with women in Brisbane, Australia, who have experienced DFV and engaged in some way with the legal system. It explores women's reasons for calling, or not calling, the police and their experiences with police between 2014 and 2017. The article highlights the role of police culture in appropriately responding to DFV. It suggests that cultural change is needed and may be facilitated by ongoing training, addressing gender balance within the police service and introducing women-only police stations. For our overseas readers, we then present the insights from this paper in Spanish, French, Portuguese, and German.

Keywords: domestic violence, family violence, police, violence against women

\section{SUMMARY}

- The Brisbane study reflects the outcomes of other studies in finding that it was usually the woman herself who called the police and women called for a variety of reasons. Many of the Brisbane participants reported that police had been respectful towards them, listened to them, worked with them and followed up on their safety. These positive views on police interactions often reflected that the woman believed she had developed a relationship with an individual police officer or station. However, significant concerns were raised by the interviewees. These included police failures to investigate or take other appropriate action, failures to take the DFV seriously and police blaming women or aligning with, or being manipulated by, abusers.

- Regardless of whether women reported negative or positive experiences with police, in general, participants' experiences were inconsistent over time. The women's comments highlight that some police responses reflect myths and misunderstandings associated with DFV and sometimes women have also internalised these myths.

- In Queensland, where this study took place, the police recruitment website includes pictures of police racing down a highway on motorbikes, leaping over fences and chasing someone or running 
towards something, under the caption: 'We don't do boring'. Understanding of the dynamics of DFV may, to some extent, be improved by encouraging realistic expectations at recruitment, and by training and specialisation. In some studies, police have expressed willingness to be trained and there is evidence that appropriate training can make a positive difference.

- Ruff reported on the positive effects of a Canadian 'train the trainer' program designed in collaboration between police, researchers and representatives from a range of government departments to help police officers respond to DFV. Police were trained to identify forms of DFV, consider the impact of DFV on children and respond to clients with diverse needs. After training Ruff discovered that police spent more time at the DFV scene, were more likely to collect evidence of DFV and were more likely to make referrals to support agencies.

- Approximately 30 per cent of constables are women, 21 per cent of non-commissioned officers are women and there is only one female member of the 18-member Queensland Police Executive. More generally, of the 44 members of various QPS committees, six are women, with three of those women being external members who are not QPS employees. With the move towards a more gender-balanced police service at the recruitment end, we may observe some changes in the way policing is conducted in Queensland. However, ensuring women have greater authority within the police service may be a more reliable way to improve the policing of DFV. Several studies have highlighted the work of the hundreds of women's police stations throughout Latin America. WPSs have been developed since the 1980s to 'enhance women's confidence in the criminal justice system, encourage reporting, prevent re-victimization and send a message to the community that gendered violence was no longer tolerated and men who abuse women will be made accountable'.

\section{HIGHLY INFLUENTIAL ARTICLE}

We used the following article as a basis of our evaluation:

Douglas, H. (2019). Policing Domestic and Family Violence. International Journal for Crime, Justice and Social Democracy, 8(2), 31-49.

This is the link to the publisher's website:

https://www.crimejusticejournal.com/article/view/1122

\section{INTRODUCTION}

Recent Australian reports have recognised the importance of police as key frontline responders to domestic and family violence (DFV) (Special Taskforce on Domestic and Family Violence in Queensland 2015, 14; Victorian Government 2016). Domestic Violence Death Review reports have identified that positive and appropriate police responses may save the lives of those who have experienced DFV (Queensland Government 2017, 23; Special Taskforce on Domestic and Family Violence in Queensland 2015,12 ). DFV is now 'ordinary' and 'pervasive' in police work (Barlow and Walklate 2018; Segrave, Wilson and Fitz-Gibbon 2018). In 2013-2014, Queensland Police responded to around 180 DFV incidents daily (Special Taskforce on Domestic and Family Violence in Queensland 2015, 1). In 2016-2017, they initiated nearly 30,000 domestic violence protection order applications, around 75 percent of the total applications initiated during the period (Queensland Police Service 2017a, 25). Over 25,000 breaches of protection orders were reported to police during 2016-2017, with 85 per cent of breach offences involving male offenders (Queensland Police Service 2017a, 20). Figures from other states also show how pervasive DFV is in police work. For example, in 2006-2007, Victorian police attended over 30,000 incidents and laid DFV-related charges in around 25 per cent of these incidents (Rollings and Taylor 2008).

While reports identify the importance of the police response, those same reports have identified that police responses to DFV have fallen short of the expectations of many victims (see Special Taskforce on Domestic and Family Violence in Queensland 2015, 13-14; Victorian Government 2016, vol. 4, 1). While 
the Queensland Not Now, Not Ever Report $(2016,14)$ recognised that some inadequate police responses may result from a lack of appropriate processes, procedures and powers, police culture was also identified as a serious issue. The Not Now Not Ever Reportmade several recommendations about how to improve police culture. These included recommendations that district domestic violence coordinators be properly allocated and resourced; a deputy commissioner position be created to increase police awareness of DFV and create positive change in the QPS; and improved training for police about DFV (Special Taskforce on Domestic and Family Violence in Queensland 2015, recs. 135, 137, 138). While these recommendations have been implemented (Queensland Government 2016, ii), it is recognised that cultural change is one of the most difficult things to effect (Segrave, Wilson and Fitz-Gibbon 2018).

Despite recognition of the importance of police in DFV responses and the perspectives of survivors of DFV in informing appropriate responses, there has been no recent exploration of DFV survivors' experiences of police response to DFV in Queensland. Drawing on interviews with 65 women in Brisbane, Australia, who experienced DFV and engaged in some way with the legal system, this article explores women's reasons for calling, or not calling, the police and the interviewees' positive and negative experiences of their engagements with police between 2014 and 2017. The paper begins with a discussion of research before exploring the study results. It concludes with some considerations for possible changes to policing in the context of DFV.

\section{CONCLUSION}

The Brisbane study reflects the outcomes of other studies in finding that it was usually the woman herself who called the police (Barrett, St Pierre and Vaillancourt 2011; Hamby 2014, 145) and women called for a variety of reasons (Barrett et al. 2017; Barrett, St Pierre and Vaillancourt 2011; Leisenring 2012; Stewart, Mangan and Hannam 2013). Many of the Brisbane participants reported that police had been respectful towards them, listened to them, worked with them and followed up on their safety. These positive views on police interactions often reflected that the woman believed she had developed a relationship with an individual police officer or station. However, significant concerns were raised by the interviewees. These included police failures to investigate or take other appropriate action (especially where the parties had children together), failures to take the DFV seriously (especially its non-physical forms) and police blaming women or aligning with, or being manipulated by, abusers. Regardless of whether women reported negative or positive experiences with police, in general, participants' experiences were inconsistent over time.

The women's comments highlight that some police responses reflect myths and misunderstandings associated with DFV and sometimes women have also internalised these myths. In particular, women's reports illustrate that some police expect that women should be able to leave violent relationships, discount complaints of coercive and controlling behaviour when thereis no physical violence and may expect victims to have certain characteristics. On this point, Segrave, Wilson and Fitz-Gibbon's (2018) interviews with Victorian police appear to reflect concerns raised by women in the Brisbane study. Their study $(2018,7)$ reported:

[o]n the whole deserving victims of [DFV] existed for officers only on a purely hypothetical plane, drowned out for the most part by a steady procession of imposters, liars and timewasters, presenting what were regarded as highly suspect claims to victim status.

While women engaged a number of strategies in response to negative or inconsistent police responses, of most concern is that some women ultimately opted out of calling the police. This concern is reflected in other research that has shown that experiences with police have implications for the woman's decision about whether to call again (Barrett et al. 2017; Goodman-Delahunty and Corbo Crehan 2015; Leisenring 2012; Li et al. 2015). Research suggests that calling the police may help reduce future violence (Xie and Lynch 2017, 359). Given that police are the frontline protectors of public safety, it is a grave situation when a woman experiencing DFV believes she can no longer rely on them. 
In his interviews with English police, Myhill $(2017,14)$ found that many police retained a 'physical violence mindset' when policing DFV, and tended to view serious incidents as physical assaults. The limited understanding of DFV exhibited by some police may be explained to some extent by the fact that the crime control model tends focused on single incidents. Stark $(2012,206)$ suggests this focus may contribute to flawed approaches to policing DFV (see Segrave, Wilson and Fitz-Gibbon 2018, 12). Recruitment materials may also play a role in entrenching the expectations of new police. In Queensland, where this study took place, the police recruitment website includes pictures of police racing down a highway on motorbikes, leaping over fences and chasing someone or running towards something, under the caption: 'We don't do boring' (Queensland Police Recruiting 2018). Understanding of the dynamics of DFV may, to some extent, be improved by encouraging realistic expectations at recruitment, and by training and specialisation. In some studies, police have expressed willingness to be trained (Horwitz et al. 2011, 623) and there is evidence that appropriate training can make a positive difference (Ruff 2012, 295). Ruff (2012) reported on the positive effects of a Canadian 'train the trainer' program designed in collaboration between police, researchers and representatives from a range of government departments to help police officers respond to DFV. Police were trained to identify forms of DFV, consider the impact of DFV on children and respond to clients with diverse needs (Ruff 2012, 287). After training Ruff $(2012,292)$ discovered that police spent more time at the DFV scene, were more likely to collect evidence of DFV and were more likely to make referrals to support agencies. Some studies have identified promising results from DFV specialty units in improving victim experiences of police, increasing the formal sanctioning of abusers and reducing DFV (Exum et al. 2014, 1003, 1024; Klein 2009; Segrave, Wilson and Fitz-Gibbon 2018). There is also US evidence to suggest that more police on the ground results in less DFV (Xie, Lauritsen and Heimer 2012, 984). In Queensland, since the Not Now, Not Ever Report (2015) was released, strategies including improved training, police specialisation and the recruitment of more police have commenced (Queensland Police Service, 2017b, a, 177). Directing women who have experienced DFV to attend specialised police units may help ensure that women experiencing DFV have a more consistently positive and appropriate experience. While allthese responses are important and research suggests they will make a difference, most have been trialled now for several years and yet rates of DFV and of DFV-related homicide remain high (Walklate, McCulloch and Fitz-Gibbon 2017). In addition, concerns about police understanding of DFV continue to be raised (Segrave, Wilson and Fitz-Gibbon 2018; Smee 2018). Perhaps we need a more radical approach.

US research suggests that there may be some differences in the way female versus male police officers do their job. Morin et al. $(2017,9,26)$ surveyed police and found that more male (35 per cent) than female (22 per cent) police reported physically struggling with or fighting a suspect who resisted arrest, and that male (30 per cent) officers were about three times more likely than female (11 per cent) officers to report firing their weapon while on duty. Research conducted in England by Andrews and Miller (2013) examined whether the presence of female police chiefs made a difference to the policing of DFV. They found that the rate of DFV arrests varied based on the authority and discretion accorded to female police officers and that higher rates of DFV arrests occurred where female chief constables were present in a police force (Andrews and Miller 2013, 1010).Importantly, they determined that 'the quality of life for women, as victims of domestic violence, can be greatly improved if there is greater representation of women within the police service' (Andrews and Miller 2013, 1011). The authors found that female police officers who had greater authority and opportunity to exercise discretion actively represented women's interests (Andrews and Miller 2013, 1010). It may be that addressing gender balance within the police service may assist in encouraging cultural change and improved responses to DFV. Queensland Police Service is overwhelmingly a male-dominated institution. The most recent statistical review undertaken by the Queensland Police Service (2017a) shows that while almost half the new recruits are women, there is an obvious gender imbalance at higher levels of the service. Approximately 30 per cent of constables are women, 21 per cent of non-commissioned officers are women and there is only one female member (six percent) of the 18-member Queensland Police Executive (Queensland Police Service 2017a). More generally, of the 44 members of various QPS committees, six (13 per cent) are women, with three of those women being external members who are not QPS employees (Queensland Police Service 2017b). With the 
move towards a more gender-balanced police service at the recruitment end, we may observe some changes in the way policing is conducted in Queensland. However, ensuring women have greater authority within the police service may be a more reliable way to improve the policing of DFV.

Several studies (Barberet and Carrington 2018; Perova and Reynolds 2015) have highlighted the work of the hundreds of women's police stations (WPSs) throughout Latin America. WPSs have been developed since the 1980 s to 'enhance women's confidence in the criminal justice system, encourage reporting, prevent re-victimization and send a message to the community that gendered violence was no longer tolerated and men who abuse women will be made accountable' (Barberet and Carrington2018, 837). WPSs operate using a variety of models, but their common features include that the stations are usually located separately from regular police stations, operators receive specialised training, and operators are able to coordinate women's access to a range of services, including services providing support through the justice system, counselling, jobs skills training and childcare (UN Women 2011, 2). UN Women $(2011,4)$ reports that WPSs have improved women's access to justice, resulting in more charges being laid against violent abusers, and the majority of WPS users surveyed reported feeling that they were listened to and, as a result, better supported and understood. Most users also perceived that WPSs contributed to a reduction in violence against women (UN Women 2011,4). Perova and Reynolds (2015) examined national mortality data in Brazil to determine the effect of WPSs on the homicide of women. They concluded that WPSs had strong effects on homicide rates among women aged 15-24 years, livingin larger metropolitan areas, resulting in a 70 per cent reduction in the average homicide rate for women in that group (Perova and Reynolds 2015, 17).

The Not Now, Not Ever Report $(2015,50)$ recognised that 'cultural and attitudinal factors ... [affect]the behaviour of the professionals within our police, justice, welfare and service provider systems when called on to respond to, and manage, domestic violence'. Police are just one of the responses to DFV, but they are an important one. This research suggests that there is still some way to go to effect the cultural change needed among police. In the Queensland context, DFV training and specialised units have been introduced and there are increasing numbers of police on the ground. However, reports of DFV and associated deaths remain persistently high (Queensland Government 2017, 35). Perhaps it is time to reconsider the police recruitment message and the representation of women in higher ranks of the police service. It might also be time to investigate the potential of women's only police stations.

\section{REFERENCES}

Ackerman, J., \& Love, T. (2014). Ethnic group differences in police notification about intimate partner violence. Violence Against Women, 20(2), 162-185. DOI: 10.1177/1077801214521327

Andrews, R., \& Johnston Miller, K. (2013). Representative bureaucracy, gender, and policing: The case of domestic violence arrests in England. Public Administration, 91(4), 998-1014. DOI: 10.1111/padm. 12002

Australasian Institute of Judicial Administration. (2018). National Domestic and Family Violence Bench Book. Retrieved April 18, 2019, from https://aija.org.au/publications/national-domestic-andfamily-violence-bench-book/

Barberet, R., \& Carrington, K. (2018). Globalizing feminist criminology: Gendered violence during peace and war. In K. Carrington, R. Hogg, J. Scott, \& M. Sozzo (Eds.), The Palgrave Book of Criminology and the Global South (pp. 821-845). London: Palgrave Macmillan. DOI: 10.1007/978-3-319-65021-0_39

Barlow, C., \& Walklate, S. (2018, January 19). Policing intimate partner violence: The 'golden thread' of discretion. Policing: A Journal of Policy and Practice. Epub ahead of print. DOI:10.1093/police/pay001

Barrett, B.J., \& St Pierre, M. (2011). Variations in women's help seeking in response to intimate partner violence: Findings from a Canadian population-based study. Violence Against Women, 17(1), 4770. DOI: $10.1177 / 1077801210394273$ 
Barrett, B.J., Peirone, A., Cheung, C.H., \& Habibov, N. (2017). Pathways to police contact for spousal violence survivors: The role of individual and neighborhood factors in survivors' reporting behaviors. Journal of Interpersonal Violence. Epub ahead of print, 1 September 2017. DOI:10.1177/0886260517729400

Barrett, B.J., St Pierre, M., \& Vaillancourt, N. (2011). Police response to intimate partner violence in Canada: Do victim characteristics matter? Women \& Criminal Justice, 21(1), 38-62. DOI: $10.1080 / 08974454.2011 .536057$

Birdsey, E., \& Snowball, L. (2013). Reporting violence to police: A survey of victims attending domestic violence services. New South Wales Bureau of Crime Statistics and Research. Retrieved April 8, 2019, from https://www.women.nsw.gov.au/_data/assets/pdf_file/0004/280912/Reporting Violence_to_the_Police_-_BOCSAR_survey.pdf

Braun, V., \& Clarke, V. (2006). Using thematic analysis in psychology. Qualitative Research in Psychology, 3(2), 77-101.https://doi.org/10.1191/1478088706qp063oa

Carrington, K. (2018, September 26-28). Where to From Here? Paper presented at Critical Criminology and Social Justice Conference. Sydney, New South Wales; University of New South Wales.

Douglas, H. (2018a). Legal systems abuse and coercive control. Criminology \& Criminal Justice, 18(1), 84-91. https://doi.org/10.1177\%2F1748895817728380

Douglas, H. (2018b). Domestic violence protection orders and their role in ensuring personal security. In K. Fitz-Gibbon, S. Walklate, J. McCulloch, \& J.M. Maher (Eds.), Intimate Partner Violence, Risk and Security: Securing Women's Lives in a Global World (pp. 216-232). London: Routledge.

Douglas, H., \& Burdon, M. (2018). Legal responses to non-consensual smartphone recordings in the context of domestic and family violence. New South Wales Law Journal, 41(1), 157-184. Retrieved April 30, 2019, from http://www.unswlawjournal.unsw.edu.au/article/legal-responsesto-non-consensual-smartphone-recordings-in-the-context-of-domestic-and-family-violence/

Douglas, H., \& Kerr, K. (2018). Domestic and family violence, reproductive coercion and the role for law. Journal of Law and Medicine, 26, 341-355.

Exum, M.L., Hartman, J.L., Friday, P.C., \& Lord, V.B. (2014). Policing domestic violence in the postSARP era: The impact of a domestic violence police unit. Crime \& Delinquency, 60(7), 9991032. DOI: $10.1177 / 0011128710382345$

Flick, U. (2007). Designing Qualitative Research. London: Sage.

Goodman-Delahunty, J., \& Corbo Crehan, A. (2015). Enhancing police responses to domestic violence incidents: Reports from client advocates in New South Wales. Violence Against Women, 22(8), 1007-1026. https://doi.org/10.1177\%2F1077801215613854

Gover, A.R., Paul, D.P., \& Dodge, M. (2011). Law enforcement officers' attitudes about domestic violence. Violence Against Women, 17(5), 619-636. DOI: 10.1177/1077801211407477

Hamby, S. (2014). Battered Women's Protective Strategies: Stronger Than You Know. New York: Oxford University Press.

Hirschel, D., \& Buzawa, E.S. (2013). The impact of offenders leaving the scene on the police decision to arrest in cases of intimate partner violence. Violence Against Women, 19(9), 1079-1103. DOI:10.1177/1077801213501843

Horwitz, S.H., Mitchell, D., LaRussa-Trott, M., Santiago, L., Pearson, J., Skiff, D.M., \& Cerulli, C. (2011). An inside view of police officers' experience with domestic violence. Journal of Family Violence, 26(8), 617-625. https://doi.org/10.1007/s10896-011-9396-y

Hoyle, C., \& Sanders, A. (2000). Police response to domestic violence: From victim choice to victim empowerment. British Journal of Criminology, 40(1), 14-36. DOI: 10.1093/bjc/40.1.14

Kelly, L., \& Westmarland, N. (2015). Naming and defining 'domestic violence': Lessons from research with violent men. Feminist Review, 112(1), 113-127. DOI: 10.1057/fr.2015.52

Kim, D. (2017). The impact of learning management systems on academic performance: Virtual Competency and student Involvement. Journal of Higher Education Theory and Practice, 17(2). Retrieved from https://articlegateway.com/index.php/JHETP/article/view/1564 
Klein, A.R. (2009). Practical implications of current domestic violence research: For law enforcement, prosecutors and judges. National Institute of Justice Special Report, June. Retrieved April 8, 2019, from https://www.ncjrs.gov/pdffiles1/nij/225722.pdf

Leisenring, A. (2011). "Whoa! They could've arrested me!”: Unsuccessful identity claims of women during police response to intimate partner violence. Qualitative Sociology, 34(2), 353-370. DOI:10.1007/s11133-011-9190-4

Leisenring, A. (2011). Victims' perceptions of police response to intimate partner violence. Journal of Police Crisis Negotiations, 12(2), 146-164. DOI: 10.1080/15332586.2012.728926.

Meyer, S. (2011). Seeking help for intimate partner violence: Victims' experiences when approaching the criminal justice system for IPV-related support and protection in an Australian jurisdiction. Feminist Criminology, 6(4), 268-290. DOI: 10.1177/1557085111414860

Morin, R., Parker, K., Stepler, R., \& Mercer, A. (2017). Behind the Badge: Amid protests and calls for reform, how police view their jobs, key issues and recent fatal encounters between blacks and police. Pew Research Centre. Retrieved April 8, 2019, from http://www.pewsocialtrends.org/2017/01/11/behind-the-badge/

Myhill, A. (2017, July). Renegotiating domestic violence: Police attitudes and decisions concerning arrest. Policing and Society, pp. 1-17. https://doi.org/10.1080/10439463.2017.1356299

Myhill, A., \& Johnson, K. (2015). Police use of discretion in response to domestic violence. Criminology \& Criminal Justice, 16(1), 3-20. DOI: 10.1177/1748895815590202

Perova, E., \& Reynolds, S. (2015). Women's Police Stations and Domestic Violence: Evidence from Brazil. World Bank Policy Research Working Paper, WPS7497. Retrieved April 8, 2019, from https://openknowledge.worldbank.org/handle/10986/23461

Powell, M., Fisher, R., \& Wright, R. (2005). Investigative interviewing in psychology and the law: An empirical perspective. In N. Brewer \& K.D. Williams (Eds.), Psychology and Law: An Empirical Perspective (pp. 11-42). New York: The Guilford Press.

Queensland Government. (2016). Queensland government response to the report of the Special Taskforce on domestic and family violence, Not Now, Not Ever: Putting an end to domestic and family violence in Queensland. Retrieved April 8, 2019, from https://www.csyw.qld.gov.au/resources/ gateway/campaigns/end-violence/qld-government-response.pdf

Queensland Government. (2017). Domestic and Family Violence Death Review and Advisory Board: 2016-2017 Annual Report. Retrieved April 8, 2019, from http://www.parliament.qld.gov.au/documents/tableOffice/TabledPapers/2017/5617T4.pdf

Queensland Police News. (2018, May 10). Officers appointed to commissioned and executive rank, my Police. Retrieved April 8, 2019, from https://mypolice.qld.gov.au/blog/2018/05/10/officersappointed-to-commissioned-and-executive-rank/

Queensland Police Recruiting. (2018). Queensland Police Recruiting. Retrieved April 8, 2019, from http://www.policerecruit.qld.gov.au/

Queensland Police Service. (2017a). 2016-2017 Annual Statistical Review. Retrieved April 8, 2019, from https://www.police.qld.gov.au/corporatedocs/reportsPublications/statisticalReview/20162017.htm

Queensland Police Service. (2017b). Annual Report 2016-2017. Retrieved April 8, 2019, from https://www.police.qld.gov.au/corporatedocs/reportsPublications/annualReport/Documents/201617/QPS-Annual-Report-2016-17.pdf

Rollings, K., \& Taylor, N. (2008). Measuring police performance in domestic and family violence. Australian Institute of Criminology Trends and Issues in Crime and Criminal Justice, 367. Retrieved April 8, 2019, from https://aic.gov.au/publications/tandi/tandi367

Ruff, L. (2012). Does training matter? Exploring police officer response to domestic dispute calls before and after training on intimate partner violence. Police Journal: Theory, Practice and Principles, 85(4), 285-300. DOI: 10.1350/pojo.2012.85.4.516 
Segrave, M., Wilson, D., \& Fitz-Gibbon, K. (2018). Policing intimate partner violence in Victoria (Australia): Examining police attitudes and the potential of specialisation. Australian \& New Zealand Journal of Criminology, 51(1), 99-116. DOI: 10.1177/0004865816679686

Smee, B. (2018, August 3). Queensland Police computer hacking: No action taken in nearly $90 \%$ of cases. The Guardian. Retrieved April 8, 2019, from https://www.theguardian.com/australianews/2018/aug/02/queensland-police-computer-hacking-no-action-taken-in-nearly-90-of-cases

Special Taskforce on Domestic and Family Violence in Queensland. (2015). Not now, not ever: Putting an end to domestic and family violence in Queensland. Retrieved April 8, 2019, from https://www.csyw.qld.gov.au/gateway/end-domestic-family-violence/about/not-now-not-everreport

Stark, E. (2012). Looking beyond domestic violence: Policing coercive control. Journal of Police Crisis Negotiations, 12(2), 199-217. DOI: 10.1080/15332586.2012.725016

Strang, H., Neyroud, P., \& Sherman, L. (2014). Tracking the evidence for a 'mythical number': Do UK domestic abuse victims suffer an average of 35 assaults before someone calls the police? Policing: A Journal of Policy and Practice, 8(2), 222-228. DOI: 10.1093/police/pau016

UN Women. (2011). Women's police stations in Latin America case study: An entry point for stopping violence and gaining access to justice (Brazil, Peru, Ecuador and Nicaragua). UN Women Security Sector Module. Retrieved April 8, 2019, from http://www.endvawnow.org/uploads/browser/files/security_wps_case_study.pdf

Victorian Government. (2016). Royal Commission into Family Violence: Summary and recommendations. Parliamentary Paper No. 132. Retrieved April 8, 2019, from http://files.rcfv.com.au/Reports/RCFV_Full_Report_Interactive.pdf

Voce, I., \& Boxall, H. (2018). Who reports domestic violence to police? A review of the evidence. Australian Institute of Criminology Trends and Issues in Crime and Criminal Justice, 559. Retrieved April 8, 2019, from https://aic.gov.au/publications/tandi/tandi559

Walklate, S., McCulloch, J., \& Fitz-Gibbon, K. (2017). Criminology, gender and security in the Australian context: Making women's lives matter. Theoretical Criminology, 23(1), 60-77. https://doi.org/10.1177\%2F1362480617719449

Westmarland, L. (2001). Gender and Policing: Sex, Power and Police Culture. Cullompton: Willan Publishing.

Xie, M., \& Lynch, J. (2017). The effects of arrest, reporting to the police, and victim services on intimate partner violence. Journal of Research in Crime and Delinquency, 54(3), 338-378. DOI:10.1177/0022427816678035

Xie, M., Lauritsen, J.L., \& Heimer, K. (2012). Intimate partner violence in US metropolitan areas: The contextual influences of police and social services. Criminology, 50(4), 961-992.

DOI:10.1111/j.1745-9125.2012.00284.x

\section{TRANSLATED VERSION: SPANISH}

Below is a rough translation of the insights presented above. This was done to give a general understanding of the ideas presented in the paper. Please excuse any grammatical mistakes and do not hold the original authors responsible for these mistakes.

\section{VERSION TRADUCIDA: ESPAÑOL}

A continuación se muestra una traducción aproximada de las ideas presentadas anteriormente. Esto se hizo para dar una comprensión general de las ideas presentadas en el documento. Por favor, disculpe cualquier error gramatical y no responsabilite a los autores originales de estos errores. 


\section{INTRODUCCIÓN}

Informes australianos recientes han reconocido la importancia de la policía como principales respondedores de primera línea a la violencia doméstica y familiar (DFV) (Special Taskforce on Domestic and Family Violence in Queensland 2015, 14; Gobierno victoriano 2016). Los informes de la Revisión de la Muerte por Violencia Doméstica han identificado que las respuestas policiales positivas y apropiadas pueden salvar las vidas de aquellos que han experimentado DFV (Gobierno de Queensland 2017, 23; Grupo de Trabajo Especial sobre Violencia Doméstica y Familiar en Queensland 2015, 12). DFV es ahora "ordinario" y "omnipresente" en el trabajo policial (Barlow y Walklate 2018; Segrave, Wilson y FitzGibbon 2018). En 2013-2014, la policía de Queensland respondió a alrededor de 180 incidentes de DFV diariamente (Grupo de Trabajo Especial sobre Violencia Doméstica y Familiar en Queensland 2015, 1). En 2016-2017, iniciaron casi 30,000 solicitudes de órdenes de protección contra la violencia doméstica, alrededor del 75 por ciento del total de solicitudes iniciadas durante el período (Servicio de Policía de Queensland 2017a, 25). Más de 25,000 violaciones de órdenes de protección fueron reportadas a la policía durante 2016-2017, con el 85 por ciento de los delitos de violación que involucran a delincuentes masculinos (Servicio de Policía de Queensland 2017a, 20). Las cifras de otros estados también muestran cuán generalizada es la DFV en el trabajo policial. Por ejemplo, en 2006-2007, la policía victoriana atendió más de 30.000 incidentes y presentó cargos relacionados con el DFV en alrededor del $25 \%$ de estos incidentes (Rollings y Taylor 2008).

Si bien los informes identifican la importancia de la respuesta policial, esos mismos informes han identificado que las respuestas policiales al DFV no han cumplido con las expectativas de muchas víctimas (véase Special Taskforce on Domestic and Family Violence in Queensland 2015, 13-14; Gobierno victoriano 2016, vol. 4, 1). Si bien el Informe Queensland Not Now, Not Ever $(2016,14)$ reconoció que algunas respuestas policiales inadecuadas pueden ser el resultado de la falta de procesos, procedimientos y poderes apropiados, la cultura policial también se identificó como un problema grave. El informe Not Now Not Ever hizo varias recomendaciones sobre cómo mejorar la cultura policial. Entre ellas figuraban las recomendaciones de que los coordinadores distritantes de violencia doméstica se asignaran y dotaran de recursos adecuados; se cree un puesto de comisionado adjunto para aumentar la conciencia de la policía sobre el DFV y crear un cambio positivo en el QPS; y la mejora de la capacitación de la policía sobre DFV (Special Taskforce on Domestic and Family Violence in Queensland 2015, recs. 135, 137, 138). Si bien estas recomendaciones se han implementado (Gobierno de Queensland 2016, ii), se reconoce que el cambio cultural es una de las cosas más difíciles de efectuar (Segrave, Wilson y Fitz-Gibbon 2018).

A pesar del reconocimiento de la importancia de la policía en las respuestas de la DFV y las perspectivas de los sobrevivientes de la DFV para informar las respuestas apropiadas, no ha habido una exploración reciente de las experiencias de los sobrevivientes de la DFV de la respuesta policial a la DFV en Queensland. Basándose en entrevistas con 65 mujeres en Brisbane, Australia, que experimentaron DFV y se involucraron de alguna manera con el sistema legal, este artículo explora las razones de las mujeres para llamar, o no llamar, a la policía y las experiencias positivas y negativas de las entrevistadas de sus compromisos con la policía entre 2014 y 2017. El documento comienza con una discusión de la investigación antes de explorar los resultados del estudio. Concluye con algunas consideraciones sobre posibles cambios en la policía en el contexto de la DFV.

\section{CONCLUSIÓN}

El estudio de Brisbane refleja los resultados de otros estudios al encontrar que generalmente era la propia mujer quien llamaba a la policía (Barrett, St Pierre y Vaillancourt 2011; Hamby 2014, 145) y las mujeres llamaron por una variedad de razones (Barrett et al. 2017; Barrett, St Pierre y Vaillancourt 2011; Leisenring 2012; Stewart, Mangan y Hannam 2013). Muchos de los participantes de Brisbane informaron que la policía había sido respetuosa con ellos, los había escuchado, trabajado con ellos y había hecho un seguimiento de su seguridad. Estas opiniones positivas sobre las interacciones policiales a menudo reflejaban que la mujer creía que había desarrollado una relación con un oficial de policía o estación de 
policía individual. Sin embargo, los entrevistados plantearon preocupaciones significativas. Estos incluyeron fallas policiales para investigar o tomar otras medidas apropiadas (especialmente cuando las partes tuvieron hijos juntas), fallas en tomar en serio el DFV (especialmente sus formas no físicas) y la policía culpando a las mujeres o alineándose con, o siendo manipulado por, abusadores. Independientemente de si las mujeres reportaron experiencias negativas o positivas con la policía, en general, las experiencias de los participantes fueron inconsistentes con el tiempo.

Los comentarios de las mujeres destacan que algunas respuestas policiales reflejan mitos y malentendidos asociados con DFV y, a veces, las mujeres también han internalizado estos mitos. En particular, los informes de las mujeres ilustran que algunos policías esperan que las mujeres puedan abandonar las relaciones violentas, descartan las denuncias de comportamiento coercitivo y controlador cuando no hay violencia física y pueden esperar que las víctimas tengan ciertas características. En este punto, las entrevistas de Segrave, Wilson y Fitz-Gibbon (2018) con la policía victoriana parecen reflejar las preocupaciones planteadas por las mujeres en el estudio de Brisbane. Su estudio $(2018,7)$ informó:

[o]n todo el merecido de [DFV] existía para los oficiales sólo en un avión puramente hipotético, ahogado en su mayor parte por una procesión constante de impostores, mentirosos y perdidores de tiempo, presentando lo que se consideraban reclamos altamente sospechosos de la condición de víctima.

Si bien las mujeres participaron en una serie de estrategias en respuesta a respuestas policiales negativas o inconsistentes, lo más preocupante es que algunas mujeres finalmente optaron por no llamar a la policía. Esta preocupación se refleja en otras investigaciones que han demostrado que las experiencias con la policía tienen implicaciones para la decisión de la mujer sobre si volver a llamar (Barrett et al. 2017; GoodmanDelahunty y Corbo Crehan 2015; Leisenring 2012; Li et al. 2015). La investigación sugiere que llamar a la policía puede ayudar a reducir la violencia futura (Xie y Lynch 2017, 359). Dado que la policía es la protectora de primera línea de la seguridad pública, es una situación grave cuando una mujer que experimenta DFV cree que ya no puede confiar en ellos.

En sus entrevistas con la policía inglesa, Myhill $(2017,14)$ encontró que muchos policías conservaban una "mentalidad de violencia física" cuando vigilaban el DFV, y tendían a ver los incidentes graves como agresiones físicas. La comprensión limitada de DFV exhibida por algunos policías puede explicarse en cierta medida por el hecho de que el modelo de control del crimen tiende a centrarse en incidentes individuales. Stark $(2012,206)$ sugiere que este enfoque puede contribuir a enfoques defectuosos para vigilar el DFV (ver Segrave, Wilson y Fitz-Gibbon 2018, 12). Los materiales de reclutamiento también pueden desempeñar un papel en el afianzamiento de las expectativas de la nueva policía. En Queensland, donde se llevó a cabo este estudio, el sitio web de reclutamiento de la policía incluye imágenes de policías corriendo por una carretera en motocicletas, saltando vallas y persiguiendo a alguien o corriendo hacia algo, bajo el título: "No hacemos aburrimiento" (Queensland Police Recruiting 2018). La comprensión de la dinámica de la DFV puede, en cierta medida, mejorarse fomentando expectativas realistas en el reclutamiento, y mediante la capacitación y la especialización. En algunos estudios, la policía ha expresado su voluntad de ser entrenada (Horwitz et al. 2011, 623) y hay evidencia de que la capacitación adecuada puede marcar una diferencia positiva (Ruff 2012, 295). Ruff (2012) informó sobre los efectos positivos de un programa canadiense de "capacitación del capacitador" diseñado en colaboración entre la policía, investigadores y representantes de una variedad de departamentos gubernamentales para ayudar a los oficiales de policía a responder al DFV. La policía fue entrenada para identificar formas de DFV, considerar el impacto de DFV en los niños y responder a clientes con diversas necesidades (Ruff 2012, 287). Después de entrenar, Ruff $(2012,292)$ descubrió que la policía pasaba más tiempo en la escena del DFV, era más propensa a recopilar evidencia de DFV y era más probable que hiciera referencias a agencias de apoyo. Algunos estudios han identificado resultados prometedores de las unidades especializadas de DFV en la mejora de las experiencias de las víctimas de la policía, el aumento de la sanción formal de los abusadores y la reducción de DFV (Exum et al. 2014, 1003, 1024; Klein 2009; Segrave, Wilson y Fitz-Gibbon 2018). También hay evidencia estadounidense que sugiere que más policía en el terreno resulta en menos DFV 
(Xie, Lauritsen y Heimer 2012, 984). En Queensland, desde que se publicó el Informe Not Now, Not Ever (2015), se han iniciado estrategias que incluyen la mejora de la capacitación, la especialización policial y el reclutamiento de más policías (Servicio de Policía de Queensland, 2017b, a, 177). Ordenar a las mujeres que han experimentado DFV que asistan a unidades policiales especializadas puede ayudar a garantizar que las mujeres que experimentan DFV tengan una experiencia más consistentemente positiva y apropiada. Si bien todas estas respuestas son importantes y la investigación sugiere que marcarán la diferencia, la mayoría se han probado ahora durante varios años y, sin embargo, las tasas de DFV y de homicidio relacionado con DFV siguen siendo altas (Walklate, McCulloch y Fitz-Gibbon 2017). Además, se siguen planteando preocupaciones sobre la comprensión policial de DFV (Segrave, Wilson y Fitz-Gibbon 2018; Smee 2018). Quizás necesitemos un enfoque más radical.

La investigación estadounidense sugiere que puede haber algunas diferencias en la forma en que las mujeres frente a los hombres policías hacen su trabajo. Morin et al. $(2017,9,26)$ encuestaron a la policía y encontraron que más policías masculinos (35 por ciento) que mujeres ( 22 por ciento) informaron haber luchado físicamente o luchar contra un sospechoso que se resistió al arresto, y que los oficiales masculinos (30 por ciento) tenían aproximadamente tres veces más probabilidades que las mujeres (11 por ciento) de informar que disparaban su arma mientras estaban de servicio. La investigación realizada en Inglaterra por Andrews y Miller (2013) examinó si la presencia de jefas de policía hizo una diferencia en la vigilancia de DFV. Encontraron que la tasa de arrestos por DFV variaba en función de la autoridad y la discreción otorgada a las mujeres oficiales de policía y que las tasas más altas de arrestos de DFV ocurrían cuando las jefas de policía estaban presentes en una fuerza policial (Andrews y Miller 2013, 1010). Es importante destacar que determinaron que "la calidad de vida de las mujeres, como víctimas de violencia doméstica, puede mejorarse en gran medida si hay una mayor representación de las mujeres dentro del servicio de policía" (Andrews y Miller 2013, 1011). Los autores encontraron que las mujeres policías que tenían mayor autoridad y oportunidad de ejercer discreción representaban activamente los intereses de las mujeres (Andrews y Miller 2013, 1010). Puede ser que abordar el equilibrio de género dentro del servicio de policía pueda ayudar a fomentar el cambio cultural y mejorar las respuestas al DFV. El Servicio de Policía de Queensland es abrumadoramente una institución dominada por hombres. La revisión estadística más reciente realizada por el Servicio de Policía de Queensland (2017a) muestra que, si bien casi la mitad de los nuevos reclutas son mujeres, existe un desequilibrio de género obvio en los niveles más altos del servicio. Aproximadamente el 30 por ciento de los agentes de policía son mujeres, el 21 por ciento de los suboficiales son mujeres y solo hay una mujer miembro (seis por ciento) del Ejecutivo de Policía de Queensland de 18 miembros (Servicio de Policía de Queensland 2017a).2Más en general, de los 44 miembros de varios comités de QPS, seis (13 por ciento) son mujeres, y tres de esas mujeres son miembros externos que no son empleados de QPS (Servicio de Policía de Queensland 2017b). Con el avance hacia un servicio de policía más equilibrado en cuanto al género al final del reclutamiento, podemos observar algunos cambios en la forma en que se lleva a cabo la policía en Queensland. Sin embargo, garantizar que las mujeres tengan mayor autoridad dentro del servicio de policía puede ser una forma más confiable de mejorar la vigilancia policial de DFV.

Varios estudios (Barberet y Carrington 2018; Perova y Reynolds 2015) han destacado el trabajo de los cientos de comisarías de mujeres (WPS) en toda América Latina. Los WPS se han desarrollado desde la década de 1980 para "mejorar la confianza de las mujeres en el sistema de justicia penal, alentar la denuncia, prevenir la revictimización y enviar un mensaje a la comunidad de que la violencia de género ya no se toleraba y que los hombres que abusan de las mujeres serán responsables" (Barberet y Carrington2018, 837). Los WPS operan utilizando una variedad de modelos, pero sus características comunes incluyen que las estaciones generalmente están ubicadas separadas de las estaciones de policía regulares, los operadores reciben capacitación especializada y los operadores pueden coordinar el acceso de las mujeres a una variedad de servicios, incluidos los servicios que brindan apoyo a través del sistema de justicia, asesoramiento, capacitación en habilidades laborales y cuidado de niños (ONU Mujeres 2011, 2). ONU Mujeres $(2011,4)$ informa que los WPS han mejorado el acceso de las mujeres a la justicia, lo que ha dado lugar a que se presenten más cargos contra los abusadores violentos, y la mayoría de los usuarios de WPS encuestados informaron sentirse escuchadas $\mathrm{y}$, como resultado, mejor apoyadas y comprendidas. La 
mayoría de los usuarios también percibieron que los WPS contribuyeron a una reducción de la violencia contra las mujeres (ONU Mujeres 2011, 4). Perova y Reynolds (2015) examinaron los datos nacionales de mortalidad en Brasil para determinar el efecto de los WPS en el homicidio de mujeres. Concluyeron que los WPS tuvieron fuertes efectos en las tasas de homicidio entre las mujeres de 15 a 24 años que viven en áreas metropolitanas más grandes, lo que resultó en una reducción del 70 por ciento en la tasa promedio de homicidios para las mujeres de ese grupo (Perova y Reynolds 2015, 17).

El Informe Not Now, Not Ever $(2015,50)$ reconoció que «los factores culturales y actitudinales [...] [afectar] el comportamiento de los profesionales dentro de nuestros sistemas de policía, justicia, bienestar y proveedores de servicios cuando se les pide que respondan y gestionen la violencia doméstica. La policía es solo una de las respuestas a DFV, pero son importantes. Esta investigación sugiere que todavía hay un camino por recorrer para lograr el cambio cultural necesario entre la policía. En el contexto de Queensland, se ha introducido la capacitación de la DFV y las unidades especializadas y hay un número cada vez mayor de policías sobre el terreno. Sin embargo, los informes de muertes asociadas a DFVand siguen siendo persistentemente altos (Gobierno de Queensland 2017, 35). Tal vez sea hora de reconsiderar el mensaje de reclutamiento de la policía y la representación de las mujeres en los rangos más altos del servicio de policía. También podría ser el momento de investigar el potencial de las comisarías de policía solo para mujeres.

\section{TRANSLATED VERSION: FRENCH}

Below is a rough translation of the insights presented above. This was done to give a general understanding of the ideas presented in the paper. Please excuse any grammatical mistakes and do not hold the original authors responsible for these mistakes.

\section{VERSION TRADUITE: FRANÇAIS}

Voici une traduction approximative des idées présentées ci-dessus. Cela a été fait pour donner une compréhension générale des idées présentées dans le document. Veuillez excuser toutes les erreurs grammaticales et ne pas tenir les auteurs originaux responsables de ces erreurs.

\section{INTRODUCTION}

Des rapports australiens récents ont reconnu l'importance de la police en tant qu'intervenant de première ligne clé en matière de violence domestique et familiale (DFV) (Special Taskforce on Domestic and Family Violence in Queensland 2015, 14; Gouvernement victorien, 2016). Les rapports de l'examen des décès dus à la violence familiale ont révélé que des réponses policières positives et appropriées peuvent sauver la vie de ceux qui ont subi une violence sexuelle et sexiste (Queensland Government 2017, 23; Special Taskforce on Domestic and Family Violence in Queensland 2015, 12). La DFV est maintenant « ordinaire » et « omniprésente » dans le travail policier (Barlow et Walklate 2018; Segrave, Wilson et FitzGibbon, 2018). En 2013-2014, la police du Queensland est intervenue quotidiennement sur environ 180 incidents de DFV (Special Taskforce on Domestic and Family Violence in Queensland 2015, 1). En 20162017, ils ont lancé près de 30000 demandes d'ordonnance de protection contre la violence domestique, soit environ 75\% du total des demandes initiées au cours de la période (Queensland Police Service 2017a, 25). Plus de 25000 infractions aux ordonnances de protection ont été signalées à la police entre 2016 et 2017, dont $85 \%$ des infractions impliquant des délinquants de sexe masculin (Queensland Police Service 2017a, 20). Les chiffres d'autres États montrent également à quel point la DFV est omniprésente dans le travail de la police. Par exemple, en 2006-2007, la police victorienne a assisté à plus de 30000 incidents et a porté des accusations liées à la DFV dans environ $25 \%$ de ces incidents (Rollings et Taylor, 2008).

Bien que les rapports identifient l'importance de l'intervention de la police, ces mêmes rapports ont révélé que les réponses de la police à la DFV n'ont pas répondu aux attentes de nombreuses victimes (voir Special Taskforce on Domestic and Family Violence in Queensland 2015, 13-14; Gouvernement victorien 2016, vol. 4, 1). Bien que le rapport Queensland Not Now, Not Ever $(2016,14)$ reconnaisse que certaines 
réponses inadéquates de la police peuvent résulter d'un manque de processus, de procédures et de pouvoirs appropriés, la culture policière a également été identifiée comme un problème grave. Le rapport Not Now Not Ever a fait plusieurs recommandations sur la façon d'améliorer la culture policière. Il s'agissait notamment de recommandations visant à ce que les coordonnateurs de district en matière de violence familiale soient correctement répartis et dotés de ressources; la création d'un poste de sous-commissaire afin de sensibiliser davantage la police à la VPH et de créer des changements positifs dans la SCP; et l'amélioration de la formation de la police sur le DFV (Special Taskforce on Domestic and Family Violence in Queensland 2015, recs. 135, 137, 138). Bien que ces recommandations aient été mises en œuvre (Queensland Government 2016, ii), il est reconnu que le changement culturel est l'une des choses les plus difficiles à mettre en œuvre (Segrave, Wilson et Fitz-Gibbon 2018).

Malgré la reconnaissance de l'importance de la police dans les interventions DFV et les points de vue des survivants de DFV dans l'information des réponses appropriées, il n'y a pas eu d'exploration récente des expériences des survivants DFV de la réponse policière à DFV dans le Queensland. S'appuyant sur des entretiens avec 65 femmes à Brisbane, en Australie, qui ont connu la DFV et se sont engagées d'une manière ou d'une autre avec le système juridique, cet article explore les raisons pour lesquelles les femmes appellent, ou n'appellent pas, la police et les expériences positives et négatives des personnes interrogées de leurs engagements avec la police entre 2014 et 2017. L'article commence par une discussion de la recherche avant d'explorer les résultats de l'étude. Il se termine par quelques considérations sur d'éventuels changements à apporter aux services de police dans le contexte de la DFV.

\section{CONCLUSION}

L'étude de Brisbane reflète les résultats d'autres études en concluant que c'était habituellement la femme elle-même qui appelait la police (Barrett, St Pierre et Vaillancourt, 2011; Hamby 2014, 145) et les femmes ont appelé pour diverses raisons (Barrett et al. 2017; Barrett, St Pierre et Vaillancourt 2011; Leisenring 2012; Stewart, Mangan et Hannam, 2013). De nombreux participants de Brisbane ont indiqué que la police avait été respectueuse envers eux, les avait écoutés, avait travaillé avec eux et avait fait un suivi de leur sécurité. Ces opinions positives sur les interactions avec la police reflétaient souvent que la femme croyait avoir développé une relation avec un agent de police ou un poste de police. Cependant, les personnes interrogées ont soulevé d'importantes préoccupations. Il s'agissait notamment de l'omission de la police d'enquêter ou de prendre d'autres mesures appropriées (en particulier lorsque les parties avaient des enfants ensemble), de l'incapacité de prendre la DFV au sérieux (en particulier ses formes non physiques) et de la police blâmant les femmes ou s'alignant sur les agresseurs ou étant manipulée par eux. Peu importe si les femmes ont signalé des expériences négatives ou positives avec la police, en général, les expériences des participants étaient incohérentes au fil du temps.

Les commentaires des femmes soulignent que certaines réponses de la police reflètent des mythes et des malentendus associés à la DFV et que parfois les femmes ont également intériorisé ces mythes. En particulier, les rapports des femmes montrent que certains policiers s'attendent à ce que les femmes puissent quitter des relations violentes, écartent les plaintes de comportement coercitif et de contrôle lorsqu'il n'y a pas de violence physique et peuvent s'attendre à ce que les victimes aient certaines caractéristiques. Sur ce point, les entretiens de Segrave, Wilson et Fitz-Gibbon (2018) avec la police victorienne semblent refléter les préoccupations soulevées par les femmes dans l'étude de Brisbane. Leur étude $(2018,7)$ a rapporté:

[o]n l'ensemble des victimes méritantes de [DFV] n'existaient pour les officiers que sur un plan purement hypothétique, noyé pour la plupart par un cortège constant d'imposteurs, de menteurs et de gaspilleurs de temps, présentant ce qui était considéré comme des revendications hautement suspectes au statut de victime.

Bien que les femmes aient adopté un certain nombre de stratégies en réponse à des réponses policières négatives ou incohérentes, ce qui est le plus préoccupant, c'est que certaines femmes ont finalement choisi de ne pas appeler la police. Cette préoccupation se reflète dans d'autres recherches qui ont montré que les 
expériences avec la police ont des répercussions sur la décision de la femme d'appeler à nouveau (Barrett et coll., 2017; Goodman-Delahunty et Corbo Crehan 2015; Leisenring 2012; Li et coll., 2015). La recherche suggère que le fait d'appeler la police peut aider à réduire la violence future (Xie et Lynch 2017, 359). Étant donné que la police est la première ligne protectrice de la sécurité publique, il s'agit d'une situation grave lorsqu'une femme victime de la DFV croit qu'elle ne peut plus compter sur elle.

Dans ses entretiens avec la police anglaise, Myhill $(2017,14)$ a constaté que de nombreux policiers conservaient un « état d'esprit de violence physique » lorsqu'ils surveillaient la DFV et avaient tendance à considérer les incidents graves comme des agressions physiques. La compréhension limitée de la DFV dont font preuve certains policiers peut s'expliquer dans une certaine mesure par le fait que le modèle de contrôle de la criminalité a tendance à se concentrer sur des incidents uniques. Stark $(2012,206)$ suggère que cette focalisation pourrait contribuer à des approches imparfaites en matière de maintien de l'ordre en matière de DFV (voir Segrave, Wilson et Fitz-Gibbon 2018, 12). Le matériel de recrutement peut également jouer un rôle dans l'enracinement des attentes des nouveaux policiers. Dans le Queensland, où cette étude a eu lieu, le site Web de recrutement de la police comprend des photos de policiers qui courent sur une autoroute à moto, sautent par-dessus les clôtures et poursuivent quelqu'un ou courent vers quelque chose, sous la légende: « Nous ne faisons pas ennuyeux » (Queensland Police Recruiting 2018). La compréhension de la dynamique de la DFV peut, dans une certaine mesure, être améliorée en encourageant des attentes réalistes lors du recrutement, ainsi que par la formation et la spécialisation. Dans certaines études, la police a exprimé sa volonté d'être formée (Horwitz et coll., 2011, 623) et il existe des preuves qu'une formation appropriée peut faire une différence positive (Ruff, 2012, 295). Ruff (2012) a fait état des effets positifs d'un programme canadien de formation des formateurs conçu en collaboration entre la police, des chercheurs et des représentants de divers ministères pour aider les policiers à réagir à la DFV. La police a été formée pour identifier les formes de DFV, examiner l'impact de la DFV sur les enfants et répondre aux clients ayant des besoins divers (Ruff 2012, 287). Après sa formation, Ruff $(2012,292)$ a découvert que la police passait plus de temps sur les lieux de la DFV, était plus susceptible de recueillir des preuves de DFV et était plus susceptible de faire des renvois à des organismes de soutien. Certaines études ont permis de dégager des résultats prometteurs des unités spécialisées de la DFV dans l'amélioration de l'expérience des victimes par la police, l'augmentation de la sanction formelle des agresseurs et la réduction de la DFV (Exum et al. 2014, 1003, 1024; Klein, 2009; Segrave, Wilson et Fitz-Gibbon, 2018). Il existe également des preuves américaines suggérant qu'un plus grand nombre de policiers sur le terrain entraîne moins de DFV (Xie, Lauritsen et Heimer, 2012, 984). Dans le Queensland, depuis la publication du rapport Not Now, Not Ever (2015), des stratégies telles que l'amélioration de la formation, la spécialisation de la police et le recrutement d'un plus grand nombre de policiers ont commencé (Queensland Police Service, 2017b, a, 177). Demander aux femmes qui ont fait l'expérience de la DFV de fréquenter des unités de police spécialisées peut aider à faire en sorte que les femmes qui en subissent la DFV aient une expérience plus positive et appropriée. Bien que toutes ces réponses soient importantes et que la recherche suggère qu'elles feront une différence, la plupart sont mises à l'essai depuis plusieurs années et pourtant, les taux d'homicides liés à la DFV et à la DFV restent élevés (Walklate, McCulloch et Fitz-Gibbon, 2017). De plus, des préoccupations concernant la compréhension de la DFV par la police continuent d'être soulevées (Segrave, Wilson et Fitz-Gibbon, 2018; Smee, 2018). Peut-être avons-nous besoin d'une approche plus radicale.

Des recherches américaines suggèrent qu'il peut y avoir des différences dans la façon dont les policiers féminins et masculins font leur travail. Morin et coll. $(2017,9,26)$ ont interrogé la police et ont constaté que plus d'hommes (35\%) que de femmes ( $22 \%$ ) ont déclaré avoir lutté physiquement avec un suspect qui résistait à son arrestation ou qui s'y est battu, et que les agents de sexe masculin (30\%) étaient environ trois fois plus susceptibles que les policières $(11 \%)$ de déclarer avoir tiré avec leur arme pendant leur service. Une recherche menée en Angleterre par Andrews et Miller (2013) a examiné si la présence de femmes chefs de police faisait une différence dans le maintien de l'ordre de DFV. Ils ont constaté que le taux d'arrestations par DFV variait en fonction de l'autorité et du pouvoir discrétionnaire accordés aux policières et que des taux plus élevés d'arrestations de DFV se produisaient lorsque des policières étaient présentes dans un corps de police (Andrews et Miller, 2013, 1010). Fait important, ils ont déterminé que « la qualité de vie des 
femmes, en tant que victimes de violence familiale, peut être grandement améliorée s'il y a une plus grande représentation des femmes au sein du service de police » (Andrews et Miller, 2013, 1011). Les auteurs ont constaté que les policières qui avaient plus d'autorité et de possibilité d'exercer leur pouvoir discrétionnaire représentaient activement les intérêts des femmes (Andrews et Miller, 2013, 1010). Il se peut que l'équilibre entre les sexes au sein du service de police puisse aider à encourager le changement culturel et à améliorer les réponses à la DFV. Le service de police du Queensland est une institution majoritairement dominée par les hommes. L'examen statistique le plus récent entrepris par le service de police du Queensland (2017a) montre que si près de la moitié des nouvelles recrues sont des femmes, il existe un déséquilibre évident entre les sexes aux niveaux supérieurs du service. Environ 30 pour cent des agents de police sont des femmes, 21 pour cent des sous-officiers sont des femmes et il n'y a qu'une seule femme membre (six pour cent) des 18 membres de l'exécutif de la police du Queensland (Queensland Police Service 2017a).2Plus généralement, sur les 44 membres des divers comités QPS, six (13 pour cent) sont des femmes, trois de ces femmes étant des membres externes qui ne sont pas des employés de QPS (Queensland Police Service 2017b). Avec l'évolution vers un service de police plus équilibré entre les sexes à la fin du recrutement, nous pouvons observer certains changements dans la façon dont les services de police sont menés dans le Queensland. Cependant, veiller à ce que les femmes aient une plus grande autorité au sein du service de police peut être un moyen plus fiable d'améliorer le maintien de l'ordre de la DFV.

Plusieurs études (Barberet et Carrington 2018; Perova et Reynolds 2015) ont mis en évidence le travail des centaines de postes de police pour femmes (WPS) à travers l'Amérique latine. Les SSR ont été élaborés depuis les années 1980 pour « renforcer la confiance des femmes dans le système de justice pénale, encourager le signalement, prévenir la victimisation à nouveau et envoyer un message à la communauté que la violence sexiste n'était plus tolérée et que les hommes qui maltraitent les femmes seront tenus responsables » (Barberet et Carrington, 2018, p. 837). Les services de police sans objet fonctionnent selon divers modèles, mais leurs caractéristiques communes sont notamment que les postes sont généralement situés séparément des postes de police ordinaires, que les opérateurs reçoivent une formation spécialisée et que les opérateurs sont en mesure de coordonner l'accès des femmes à une gamme de services, $\mathrm{y}$ compris les services de soutien par l'intermédiaire du système judiciaire, le conseil, la formation professionnelle et la garde d'enfants (ONU Femmes 2011, 2). ONU Femmes $(2011,4)$ rapporte que les services de libre-état ont amélioré l'accès des femmes à la justice, ce qui a entraîné davantage d'accusations contre les agresseurs violents, et la majorité des utilisateurs des services de libre-service interrogés ont déclaré avoir le sentiment d'être écoutés et, par conséquent, mieux soutenus et compris. La plupart des utilisateurs ont également perçu que les SSR contribuaient à réduire la violence à l'égard des femmes (ONU Femmes 2011, 4). Perova et Reynolds (2015) ont examiné les données nationales sur la mortalité au Brésil pour déterminer l'effet des WPS sur les homicides de femmes. Ils ont conclu que les SSR avaient de fortes incidences sur les taux d'homicides chez les femmes âgées de 15 à 24 ans vivant dans de plus grandes régions métropolitaines, ce qui a entraîné une réduction de $70 \%$ du taux moyen d'homicides chez les femmes de ce groupe (Perova et Reynolds, 2015, p. 17).

Le rapport Not Now, Not Ever $(2015,50)$ a reconnu que « les facteurs culturels et comportementaux [...] [affecter] le comportement des professionnels au sein de nos systèmes de police, de justice, d'aide sociale et de fournisseurs de services lorsqu'ils sont appelés à réagir et à gérer la violence domestique $»$. La police n'est qu'une des réponses à la DFV, mais elle est importante. Cette recherche suggère qu'il reste encore du chemin à parcourir pour apporter le changement culturel nécessaire au sein de la police. Dans le contexte du Queensland, des unités de formation et des unités spécialisées ont été introduites et il y a de plus en plus de policiers sur le terrain. Cependant, les rapports de décès associés à la DFVand restent constamment élevés (Queensland Government 2017,35). Il est peut-être temps de reconsidérer le message de recrutement de la police et la représentation des femmes dans les échelons supérieurs des services de police. Il serait peut-être également temps d'enquêter sur le potentiel des postes de police réservés aux femmes. 


\section{TRANSLATED VERSION: GERMAN}

Below is a rough translation of the insights presented above. This was done to give a general understanding of the ideas presented in the paper. Please excuse any grammatical mistakes and do not hold the original authors responsible for these mistakes.

\section{ÜBERSETZTE VERSION: DEUTSCH}

Hier ist eine ungefähre Übersetzung der oben vorgestellten Ideen. Dies wurde getan, um ein allgemeines Verständnis der in dem Dokument vorgestellten Ideen zu vermitteln. Bitte entschuldigen Sie alle grammatikalischen Fehler und machen Sie die ursprünglichen Autoren nicht für diese Fehler verantwortlich.

\section{EINLEITUNG}

Jüngste australische Berichte haben die Bedeutung der Polizei als wichtige Frontline-Responder bei häuslicher und familiärer Gewalt (DFV) anerkannt (Special Taskforce on Domestic and Family Violence in Queensland 2015, 14; Viktorianische Regierung 2016). Berichte des Domestic Violence Death Review haben ergeben, dass positive und angemessene Polizeireaktionen das Leben derjenigen retten können, die DFV erlebt haben (Queensland Government 2017, 23; Special Taskforce on Domestic and Family Violence in Queensland 2015, 12). DFV ist jetzt "gewöhnlich" und "allgegenwärtig" in der Polizeiarbeit (Barlow und Walklate 2018; Segrave, Wilson und Fitz-Gibbon 2018). In den Jahren 2013-2014 reagierte die Polizei von Queensland täglich auf rund 180 DFV-Vorfälle (Special Taskforce on Domestic and Family Violence in Queensland 2015, 1). In den Jahren 2016-2017 initiierten sie fast 30.000 Anträge auf Schutz vor häuslicher Gewalt, rund 75 Prozent der gesamten Anträge, die in diesem Zeitraum initiiert wurden (Queensland Police Service 2017a, 25). Über 25.000 Verstöße gegen Schutzanordnungen wurden der Polizei zwischen 2016 und 2017 gemeldet, wobei 85 Prozent der Verstöße männliche Straftäter betrafen (Queensland Police Service 2017a, 20). Zahlen aus anderen Bundesländern zeigen auch, wie weit verbreitet DFV in der Polizeiarbeit ist. Zum Beispiel nahm die viktorianische Polizei in den Jahren 2006-2007 an über 30.000 Vorfällen teil und erhob bei rund 25 Prozent dieser Vorfälle DFV-bezogene Anklagen (Rollings und Taylor 2008).

Während Berichte die Bedeutung der polizeilichen Reaktion auffallen, haben dieselben Berichte festgestellt, dass die Reaktionen der Polizei auf DFV hinter den Erwartungen vieler Opfer zurückblieben (siehe Special Taskforce on Domestic and Family Violence in Queensland 2015, 13-14; Victorian Government 2016, Bd. 4, 1). Während der Queensland Not Now, Not Ever Report $(2016,14)$ anerkannte, dass einige unzureichende Polizeireaktionen auf einen Mangel an geeigneten Prozessen, Verfahren und Befugnissen zurückzuführen sein können, wurde auch die Polizeikultur als ernstes Problem identifiziert. Der Not Now Not Ever Report gab mehrere Empfehlungen, wie die Polizeikultur verbessert werden kann. Dazu gehörten Empfehlungen, dass die Koordinatoren für häusliche Gewalt in den Distrikten ordnungsgemäß zugewiesen und mit Ressourcen ausgestattet werden; schaffung einer stellvertretenden Kommissarsstelle, um das Bewusstsein der Polizei für DFV zu schärfen und positive Veränderungen in der QPS herbeizuführen; und verbesserte Schulungen für Polizisten über DFV (Special Taskforce on Domestic and Family Violence in Queensland 2015, recs. 135, 137, 138). Obwohl diese Empfehlungen umgesetzt wurden (Queensland Government 2016, ii), wird anerkannt, dass der kulturelle Wandel eines der schwierigsten Dinge ist (Segrave, Wilson und Fitz-Gibbon 2018).

Trotz der Anerkennung der Bedeutung der Polizei bei DFV-Antworten und der Perspektiven von Überlebenden von DFV bei der Information geeigneter Reaktionen wurden die Erfahrungen von DFVÜberlebenden mit der Reaktion der Polizei auf DFV in Queensland in jüngster Zeit nicht untersucht. Basierend auf Interviews mit 65 Frauen in Brisbane, Australien, die DFV erlebt und sich in irgendeiner Weise mit dem Rechtssystem beschäftigt haben, untersucht dieser Artikel die Gründe von Frauen, die Polizei anzurufen oder nicht anzurufen, und die positiven und negativen Erfahrungen der Befragten mit 
ihren Engagements bei der Polizei zwischen 2014 und 2017. Das Papier beginnt mit einer Diskussion der Forschung, bevor die Studienergebnisse untersucht werden. Er schließt mit einigen Überlegungen zu möglichen Änderungen der Polizeiarbeit im Rahmen der DFV.

\section{SCHLUSSFOLGERUNG}

Die Brisbane-Studie spiegelt die Ergebnisse anderer Studien wider, in denen festgestellt wurde, dass es in der Regel die Frau selbst war, die die Polizei rief (Barrett, St. Pierre und Vaillancourt 2011; Hamby 2014, 145) und Frauen aus verschiedenen Gründen (Barrett et al. 2017; Barrett, St. Pierre und Vaillancourt 2011; Leisenring 2012; Stewart, Mangan und Hannam 2013). Viele der Brisbane-Teilnehmer berichteten, dass die Polizei ihnen gegenüber respektvoll gewesen sei, ihnen zugehört, mit ihnen zusammengearbeitet und ihre Sicherheit verfolgt habe. Diese positiven Ansichten über polizeiliche Interaktionen spiegelten oft wider, dass die Frau glaubte, eine Beziehung zu einem einzelnen Polizeibeamten oder einer einzelnen Wache entwickelt zu haben. Die Befragten äußerten jedoch erhebliche Bedenken. Dazu gehörten das Versäumnis der Polizei, zu ermitteln oder andere geeignete Maßnahmen zu ergreifen (insbesondere wenn die Parteien gemeinsam Kinder hatten), Versäumnisse, den DFV ernst zu nehmen (insbesondere seine nicht-physischen Formen) und die Polizei, die Frauen beschuldigte oder sich mit Tätern abbürdete oder von ihnen manipuliert wurde. Unabhängig davon, ob Frauen negative oder positive Erfahrungen mit der Polizei berichteten, waren die Erfahrungen der Teilnehmer im Allgemeinen im Laufe der Zeit inkonsistent.

Die Kommentare der Frauen heben hervor, dass einige Reaktionen der Polizei Mythen und Missverständnisse im Zusammenhang mit DFV widerspiegeln und manchmal haben Frauen diese Mythen auch verinnerlicht. Insbesondere zeigen Frauenberichte, dass einige Polizisten erwarten, dass Frauen in der Lage sein sollten, gewalttätige Beziehungen zu verlassen, Beschwerden über Zwang und kontrollierendes Verhalten zu vernachlässigen, wenn es keine körperliche Gewalt gibt, und erwarten können, dass Opfer bestimmte Merkmale haben. In diesem Punkt scheinen die Interviews von Segrave, Wilson und Fitz-Gibbon (2018) mit der viktorianischen Polizei die Bedenken von Frauen in der Brisbane-Studie widerzuspiegeln. Ihre Studie $(2018,7)$ berichtete:

Die ganzen verdienten Opfer von [DFV] existierten für Offiziere nur auf einer rein hypothetischen Ebene, die größtenteils von einer stetigen Prozession von Betrügern, Lügnern und Zeitverschwendern übertönt wurde, die als höchst verdächtige Ansprüche auf den Opferstatus galten.

Während Frauen eine Reihe von Strategien als Reaktion auf negative oder inkonsistente Reaktionen der Polizei einbewätigten, ist es am besorgniserregendsten, dass einige Frauen sich letztendlich gegen den Polizeiruf entschieden haben. Diese Besorgnis spiegelt sich in anderen Untersuchungen wider, die gezeigt haben, dass Erfahrungen mit der Polizei Auswirkungen auf die Entscheidung der Frau haben, ob sie erneut anrufen soll (Barrett et al. 2017; Goodman-Delahunty und Corbo Crehan 2015; Leisenring 2012; Li et al. 2015). Forschungsergebnisse deuten darauf hin, dass der Anruf der Polizei dazu beitragen kann, zukünftige Gewalt zu reduzieren (Xie und Lynch 2017, 359). Angesichts der Tatsache, dass die Polizei die Beschützer der öffentlichen Sicherheit an vorderster Front ist, ist es eine ernste Situation, wenn eine Frau, die DFV erlebt, glaubt, sich nicht mehr auf sie verlassen zu können.

In seinen Interviews mit der englischen Polizei stellte Myhill $(2017,14)$ fest, dass viele Polizisten bei der Überwachung von DFV eine "körperliche Gewaltmentalität" beibehielten und dazu neigten, schwere Vorfälle als körperliche Übergriffe zu betrachten. Das begrenzte Verständnis von DFV, das von einigen Polizisten gezeigt wird, kann bis zu einem gewissen Grad durch die Tatsache erklärt werden, dass sich das Verbrechenskontrollmodell tendenziell auf einzelne Vorfälle konzentriert. Stark $(2012,206)$ schlägt vor, dass dieser Fokus zu fehlerhaften Ansätzen bei der Überwachung von DFV beitragen könnte (siehe Segrave, Wilson und Fitz-Gibbon 2018, 12). Rekrutierungsmaterialien können auch eine Rolle dabei spielen, die Erwartungen an neue Polizisten zu verankern. In Queensland, wo diese Studie stattfand, enthält die Website zur Rekrutierung der Polizei Bilder von Polizisten, die mit Motorrädern eine Autobahn 
hinunterrasen, über Zäune springen und jemanden jagen oder auf etwas zulaufen, unter der Überschrift: "Wir machen nicht langweilig" (Queensland Police Recruiting 2018). Das Verständnis der Dynamik der DFV kann bis zu einem gewissen Grad verbessert werden, indem realistische Erwartungen bei der Einstellung sowie durch Ausbildung und Spezialisierung gefördert werden. In einigen Studien haben Polizisten ihre Bereitschaft bekundet, ausgebildet zu werden (Horwitz et al. 2011, 623) und es gibt Hinweise darauf, dass eine angemessene Ausbildung einen positiven Unterschied machen kann (Ruff 2012, 295). Ruff (2012) berichtete über die positiven Auswirkungen eines kanadischen "Train the Trainer"Programms, das in Zusammenarbeit zwischen Polizei, Forschern und Vertretern verschiedener Regierungsabteilungen entwickelt wurde, um Polizeibeamten zu helfen, auf DFV zu reagieren. Die Polizei wurde geschult, um Formen von DFV zu identifizieren, die Auswirkungen von DFV auf Kinder zu berücksichtigen und auf Kunden mit unterschiedlichen Bedürfnissen einzugehen (Ruff 2012, 287). Nach dem Training entdeckte Ruff $(2012,292)$, dass die Polizei mehr Zeit am DFV-Tatort verbrachte, eher Beweise für DFV sammelte und eher Überweisungen an Unterstützungsagenturen vorngab. Einige Studien haben vielversprechende Ergebnisse von DFV-Spezialeinheiten zur Verbesserung der Opfererfahrungen der Polizei, zur Erhöhung der formellen Sanktionierung von Tätern und zur Verringerung der DFV identifiziert (Exum et al. 2014, 1003, 1024; Klein 2009; Segrave, Wilson und Fitz-Gibbon 2018). Es gibt auch US-Beweise, die darauf hindeuten, dass mehr Polizei vor Ort zu weniger DFV führt (Xie, Lauritsen und Heimer 2012, 984). In Queensland haben seit der Veröffentlichung des Not Now, Not Ever Report (2015) Strategien wie verbesserte Ausbildung, Polizeispezialisierung und die Einstellung von mehr Polizisten begonnen (Queensland Police Service, 2017b, a, 177). Die Anweisen von Frauen, die DFVErfahrung haben, an spezialisierten Polizeieinheiten teilzunehmen, kann dazu beitragen, dass Frauen, die DFV erleben, eine durchweg positivere und angemessenere Erfahrung machen. Während all diese Antworten wichtig sind und die Forschung darauf hindeutet, dass sie einen Unterschied machen werden, werden die meisten seit mehreren Jahren getestet, und dennoch bleiben die Raten von DFV und DFVbezogenen Tötungsdelikten hoch (Walklate, McCulloch und Fitz-Gibbon 2017). Darüber hinaus werden weiterhin Bedenken hinsichtlich des polizeilichen Verständnisses von DFV geäußert (Segrave, Wilson und Fitz-Gibbon 2018; Smee 2018). Vielleicht brauchen wir einen radikaleren Ansatz.

US-Untersuchungen deuten darauf hin, dass es einige Unterschiede in der Art und Weise geben kann, wie weibliche und männliche Polizisten ihre Arbeit erledigen. Morin et al. $(2017,9,26)$ befragten die Polizei und fanden heraus, dass mehr männliche (35 Prozent) als weibliche (22 Prozent) Polizisten berichteten, dass sie körperlich mit einem Verdächtigen kämpften oder ihn bekämpften, der sich der Festnahme widersetzte, und dass männliche (30 Prozent) Beamte etwa dreimal häufiger als weibliche (11 Prozent) Beamte berichteten, dass sie ihre Waffe während des Dienstes abfeuerten. Eine in England von Andrews und Miller (2013) durchgeführte Studie untersuchte, ob die Anwesenheit weiblicher Polizeichefs einen Unterschied für die Polizeiarbeit von DFV machte. Sie fanden heraus, dass die Rate der DFVVerhaftungen aufgrund der Autorität und des Ermessens, die weiblichen Polizeibeamten eingeräumt wurden, variierte und dass höhere Raten von DFV-Verhaftungen auftraten, wenn weibliche Chief Constables in einer Polizeitruppe anwesend waren (Andrews und Miller 2013, 1010). Wichtig ist, dass sie feststellten, dass "die Lebensqualität von Frauen als Opfer häuslicher Gewalt erheblich verbessert werden kann, wenn Frauen im Polizeidienst stärker vertreten sind" (Andrews und Miller 2013, 1011). Die Autoren fanden heraus, dass weibliche Polizeibeamte, die mehr Autorität und Möglichkeit zur Ausübung von Diskretion hatten, aktiv die Interessen von Frauen vertraten (Andrews und Miller 2013, 1010). Es kann sein, dass die Berücksichtigung eines ausgewogenen Geschlechterverhältnisses innerhalb des Polizeidienstes dazu beitragen kann, kulturelle Veränderungen und verbesserte Reaktionen auf DFV zu fördern. Der Queensland Police Service ist überwiegend eine von Männern dominierte Institution. Die jüngste statistische Überprüfung des Queensland Police Service (2017a) zeigt, dass zwar fast die Hälfte der neuen Rekruten Frauen sind, es jedoch ein offensichtliches Ungleichgewicht zwischen den Geschlechtern auf höheren Ebenen des Dienstes gibt. Etwa 30 Prozent der Constables sind Frauen, 21 Prozent der Unteroffiziere sind Frauen und es gibt nur ein weibliches Mitglied (sechs Prozent) der 18-köpfigen Queensland Police Executive (Queensland Police Service 2017a).2Mehr sind von den 44 Mitgliedern verschiedener QPS-Komitees sechs (13 Prozent) Frauen, wobei drei dieser Frauen externe Mitglieder sind, 
die keine QPS-Mitarbeiter sind (Queensland Police Service 2017b). Mit dem Übergang zu einem ausgewogeneren Polizeidienst mit mehr Geschlechtern am Ende der Rekrutierung können wir einige Veränderungen in der Art und Weise beobachten, wie polizeiliche Maßnahmen in Queensland durchgeführt werden. Sicherzustellen, dass Frauen mehr Autorität innerhalb des Polizeidienstes haben, kann jedoch ein zuverlässigerer Weg sein, um die Polizeiarbeit des DFV zu verbessern.

Mehrere Studien (Barberet und Carrington 2018; Perova und Reynolds 2015) haben die Arbeit von Hunderten von Frauenpolizeistationen (WPSs) in ganz Lateinamerika hervorgehoben. WPS wurden seit den 1980er Jahren entwickelt, um "das Vertrauen von Frauen in das Strafjustizsystem zu stärken, die Berichterstattung $\mathrm{zu}$ fördern, eine erneute Viktimisierung $\mathrm{zu}$ verhindern und der Gemeinschaft $\mathrm{zu}$ vermitteln, dass geschlechtsspezifische Gewalt nicht mehr toleriert wird und Männer, die Frauen missbrauchen, zur Rechenschaft ziehen" (Barberet und Carrington2018, 837). WPS arbeiten mit einer Vielzahl von Modellen, aber ihre gemeinsamen Merkmale sind, dass sich die Stationen in der Regel getrennt von regulären Polizeistationen befinden, die Betreiber eine spezielle Ausbildung erhalten und die Betreiber in der Lage sind, den Zugang von Frauen zu einer Reihe von Dienstleistungen zu koordinieren, einschließlich Dienstleistungen, die Unterstützung durch das Justizsystem, Beratung, Ausbildung von Beruflichen und Kinderbetreuung bieten (UN Women 2011, 2). UN Women (2011,4) berichtet, dass WPS den Zugang von Frauen zur Justiz verbessert haben, was dazu geführt hat, dass mehr Anklagen gegen gewalttätige Täter erhoben wurden, und die Mehrheit der befragten WPS-Nutzer gab an, sich angehört und infolgedessen besser unterstützt und verstanden zu fühlen. Die meisten Nutzer nahmen auch wahr, dass WPSs zu einer Verringerung der Gewalt gegen Frauen beitrugen (UN Women 2011, 4). Perova und Reynolds (2015) untersuchten nationale Mortalitätsdaten in Brasilien, um die Wirkung von WPSs auf den Mord an Frauen zu bestimmen. Sie kamen zu dem Schluss, dass WPS starke Auswirkungen auf die Mordraten bei Frauen im Alter von 15 bis 24 Jahren hatten, die in größeren Ballungsräumen lebten, was zu einer 70-prozentigen Verringerung der durchschnittlichen Mordrate für Frauen in dieser Gruppe führte (Perova und Reynolds 2015, 17).

Der Not Now, Not Ever Report $(2015,50)$ erkannte an, dass "kulturelle und einstellungsschwellische Faktoren ... das Verhalten der Fachkräfte in unseren Polizei-, Justiz-, Sozial- und Dienstleistungssystemen beeinflussen, wenn sie aufgefordert werden, auf häusliche Gewalt zu reagieren und sie zu bewältigen." Die Polizei ist nur eine der Antworten auf DFV, aber sie sind eine wichtige. Diese Forschung legt nahe, dass es noch einen Weg zu gehen gibt, um den kulturellen Wandel herbeizuführen, der bei der Polizei erforderlich ist. Im Kontext von Queensland wurden DFV-Ausbildungs- und Spezialeinheiten eingeführt, und es gibt immer mehr Polizisten vor Ort. Die Berichte über DFVand-assoziierte Todesfälle bleiben jedoch anhaltend hoch (Queensland Government 2017, 35). Vielleicht ist es an der Zeit, die Botschaft der Polizeirekrutierung und die Vertretung von Frauen in höheren Rängen des Polizeidienstes zu überdenken. Es könnte auch an der Zeit sein, das Potenzial von Polizeistationen nur für Frauen zu untersuchen.

\section{TRANSLATED VERSION: PORTUGUESE}

Below is a rough translation of the insights presented above. This was done to give a general understanding of the ideas presented in the paper. Please excuse any grammatical mistakes and do not hold the original authors responsible for these mistakes.

\section{VERSÃO TRADUZIDA: PORTUGUÊS}

Aqui está uma tradução aproximada das ideias acima apresentadas. Isto foi feito para dar uma compreensão geral das ideias apresentadas no documento. Por favor, desculpe todos os erros gramaticais e não responsacule os autores originais responsáveis por estes erros. 


\section{INTRODUÇÃO}

Relatórios recentes australianos reconheceram a importância da polícia como principais respondentes da linha de frente para a violência doméstica e familiar (DFV) (Força-Tarefa Especial sobre Violência Doméstica e Familiar em Queensland 2015, 14; Governo Vitoriano 2016). Os relatórios da Revista de Morte por Violência Doméstica identificaram que respostas policiais positivas e apropriadas podem salvar a vida daqueles que passaram pelo DFV (Governo de Queensland 2017, 23; Força-Tarefa Especial sobre Violência Doméstica e Familiar em Queensland 2015, 12). DFV agora é 'ordinária' e 'difundida' no trabalho policial (Barlow e Walklate 2018; Segrave, Wilson e Fitz-Gibbon 2018). Em 2013-2014, a polícia de Queensland respondeu a cerca de 180 incidentes de DFV diariamente (Força Tarefa Especial sobre Violência Doméstica e Familiar em Queensland 2015, 1). Entre 2016 e 2017, eles iniciaram cerca de 30.000 pedidos de ordem de proteção à violência doméstica, cerca de $75 \%$ do total de pedidos iniciados durante o período (Serviço de Polícia de Queensland 2017a, 25). Mais de 25.000 violações de ordens de proteção foram relatadas à polícia durante 2016-2017, com 85\% dos crimes de violação envolvendo criminosos do sexo masculino (Serviço de Polícia de Queensland 2017a, 20). Dados de outros estados também mostram como o DFV é difundido no trabalho policial. Por exemplo, entre 2006 e 2007, a polícia vitoriana atendeu mais de 30.000 incidentes e apresentou acusações relacionadas ao DFV em cerca de $25 \%$ desses incidentes (Rollings e Taylor 2008).

Embora os relatos identifiquem a importância da resposta da polícia, esses mesmos relatórios identificaram que as respostas da polícia ao DFV ficaram aquém das expectativas de muitas vítimas (ver Força-Tarefa Especial sobre Violência Doméstica e Familiar em Queensland 2015, 13-14; Governo Vitoriano 2016, vol. 4, 1). Embora o Queensland Not Now, Not Ever Report $(2016,14)$ tenha reconhecido que algumas respostas policiais inadequadas podem resultar da falta de processos, procedimentos e poderes adequados, a cultura policial também foi identificada como um problema sério. O Not Now Not Ever Fez várias recomendações sobre como melhorar a cultura policial. Estas incluíam recomendações para que os coordenadores distritais de violência doméstica sejam devidamente alocados e com recursos; uma posição de vice-comissário será criada para aumentar a conscientização policial sobre o DFV e criar mudanças positivas no QPS; e melhorou o treinamento para a polícia sobre o DFV (Força-Tarefa Especial de Violência Doméstica e Familiar em Queensland 2015, recs. 135, 137, 138). Embora essas recomendações tenham sido implementadas (Governo de Queensland 2016, ii), reconhece-se que a mudança cultural é uma das coisas mais difíceis de realizar (Segrave, Wilson e Fitz-Gibbon 2018).

Apesar do reconhecimento da importância da polícia nas respostas do DFV e das perspectivas dos sobreviventes do DFV em informar respostas apropriadas, não houve exploração recente das experiências dos sobreviventes do DFV de resposta policial ao DFV em Queensland. Com base em entrevistas com 65 mulheres em Brisbane, Austrália, que vivenciaram o DFV e se envolveram de alguma forma com o sistema jurídico, este artigo explora as razões das mulheres para chamar, ou não, as experiências positivas e negativas dos entrevistados de seus compromissos com a polícia entre 2014 e 2017. O trabalho começa com uma discussão de pesquisa antes de explorar os resultados do estudo. Conclui-se com algumas considerações sobre possíveis mudanças no policiamento no contexto do DFV.

\section{CONCLUSÃO}

O estudo de Brisbane reflete os resultados de outros estudos ao descobrir que geralmente foi a própria mulher que chamou a polícia (Barrett, St Pierre e Vaillancourt 2011; Hamby 2014, 145) e mulheres pediram uma variedade de razões (Barrett et al. 2017; Barrett, St Pierre e Vaillancourt 2011; Leisenring 2012; Stewart, Mangan e Hannam 2013). Muitos dos participantes de Brisbane relataram que a polícia tinha sido respeitosa com eles, ouviu-os, trabalhou com eles e seguiu em sua segurança. Essas visões positivas sobre as interações policiais frequentemente refletiam que a mulher acreditava ter desenvolvido um relacionamento com um policial ou delegacia individual. No entanto, preocupações significativas foram levantadas pelos entrevistados. Estes incluíram falhas policiais para investigar ou tomar outras medidas apropriadas (especialmente quando as partes tinham filhos juntos), falhas em levar o DFV a sério 
(especialmente suas formas não físicas) e a polícia culpando as mulheres ou alinhando-se com, ou sendo manipulada por abusadores. Independentemente de as mulheres relataram experiências negativas ou positivas com a polícia, em geral, as experiências dos participantes foram inconsistentes ao longo do tempo.

Os comentários das mulheres destacam que algumas respostas policiais refletem mitos e malentendidos associados ao DFV e, às vezes, as mulheres também internalizaram esses mitos. Em particular, os relatos das mulheres ilustram que alguns policiais esperam que as mulheres possam sair de relações violentas, descontar queixas de comportamento coercitivo e controlador quando não há violência física e podem esperar que as vítimas tenham certas características. Neste ponto, as entrevistas de Segrave, Wilson e Fitz-Gibbon (2018) com a polícia vitoriana parecem refletir preocupações levantadas pelas mulheres no estudo de Brisbane. O estudo $(2018,7)$ relatou:

[o]n toda a vítima merecedora de [DFV] existia para oficiais apenas em um avião puramente hipotético, afogado na maior parte por uma procissão constante de impostores, mentirosos e timewasters, apresentando o que eram considerados como reivindicações altamente suspeitas ao status de vítima.

Enquanto as mulheres engajaram uma série de estratégias em resposta a respostas negativas ou inconsistentes da polícia, a maior preocupação é que algumas mulheres finalmente optaram por deixar de chamar a polícia. Essa preocupação se reflete em outras pesquisas que mostraram que experiências com a polícia têm implicações para a decisão da mulher sobre se deve ligar novamente (Barrett et al. 2017; Goodman-Delahunty e Corbo Crehan 2015; Leisenring 2012; Li et al. 2015). Pesquisas sugerem que chamar a polícia pode ajudar a reduzir a violência futura (Xie e Lynch 2017, 359). Dado que a polícia é a protetora da segurança pública, é uma situação grave quando uma mulher que vive a DFV acredita que não pode mais confiar neles.

Em suas entrevistas à polícia inglesa, Myhill $(2017,14)$ descobriu que muitos policiais mantinham uma "mentalidade de violência física" ao policiar o DFV, e tendia a ver incidentes graves como agressões físicas. O entendimento limitado do DFV exibido por alguns policiais pode ser explicado, em certa medida, pelo fato de que o modelo de controle do crime tende a se concentrar em incidentes únicos. Stark $(2012,206)$ sugere que esse foco pode contribuir para abordagens falhas no policiamento do DFV (ver Segrave, Wilson e Fitz-Gibbon 2018, 12). Os materiais de recrutamento também podem desempenhar um papel no entrincheiramento das expectativas de novas polícias. Em Queensland, onde este estudo ocorreu, o site de recrutamento da polícia inclui fotos da polícia correndo por uma rodovia em motocicletas, pulando cercas e perseguindo alguém ou correndo em direção a algo, sob a legenda: "Nós não fazemos chato" (Queensland Police Recruiting 2018). A compreensão da dinâmica do DFV pode, em certa medida, ser melhorada incentivando expectativas realistas no recrutamento e por treinamento e especialização. Em alguns estudos, a polícia tem expressado vontade de ser treinada (Horwitz et al. 2011, 623) e há evidências de que o treinamento adequado pode fazer uma diferença positiva (Ruff 2012, 295). Ruff (2012) relatou os efeitos positivos de um programa canadense de "treinar o treinador" projetado em colaboração entre a polícia, pesquisadores e representantes de uma série de departamentos governamentais para ajudar os policiais a responder ao DFV. A polícia foi treinada para identificar formas de DFV, considerar o impacto do DFV nas crianças e responder a clientes com necessidades diversas (Ruff 2012, 287). Após o treinamento, Ruff $(2012,292)$ descobriu que a polícia passava mais tempo na cena do DFV, eram mais propensas a coletar evidências de DFV e eram mais propensas a fazer encaminhamentos para agências de apoio. Alguns estudos identificaram resultados promissores das unidades especializadas do DFV na melhoria das experiências das vítimas da polícia, aumentando a sanção formal de abusadores e reduzindo o DFV (Exum et al. 2014, 1003, 1024; Klein 2009; Segrave, Wilson e Fitz-Gibbon 2018). Há também evidências dos EUA que sugerem que mais policiais no terreno resultam em menos DFV (Xie, Lauritsen e Heimer 2012, 984). Em Queensland, desde que o Not Now, Not Ever Report(2015) foi lançado, estratégias que incluem melhor treinamento, especialização policial e recrutamento de mais policiais começaram (Serviço de Polícia de Queensland, 2017b, a, 177). Orientar mulheres que vivenciaram o DFV a frequentar unidades policiais especializadas pode ajudar a garantir que as mulheres que experimentam o DFV tenham uma experiência mais 
consistentemente positiva e apropriada. Embora todas essas respostas sejam importantes e pesquisas sugerem que farão a diferença, a maioria já foi testada há vários anos e, no entanto, as taxas de DFV e de homicídios relacionados ao DFV permanecem altas (Walklate, McCulloch e Fitz-Gibbon 2017). Além disso, as preocupações com o entendimento policial da DFV continuam a ser levantadas (Segrave, Wilson e Fitz-Gibbon 2018; Smee 2018). Talvez precisemos de uma abordagem mais radical.

Pesquisas dos EUA sugerem que pode haver algumas diferenças na forma como policiais do sexo feminino versus masculino fazem seu trabalho. Morin et al. $(2017,9$, 26) entrevistaram a polícia e descobriram que mais policiais do sexo masculino (35\%) do que mulheres (22 por cento) relataram ter lutado fisicamente ou lutado contra um suspeito que resistiu à prisão, e que os oficiais do sexo masculino (30\%) eram cerca de três vezes mais propensos do que os oficiais do sexo feminino (11\%) a relatar o disparo de sua arma durante o serviço. Uma pesquisa realizada na Inglaterra por Andrews e Miller (2013) analisou se a presença de chefes de polícia femininas fez diferença para o policiamento do DFV. Eles descobriram que a taxa de prisões do DFV variou com base na autoridade e discrição concedida às mulheres policiais e que as maiores taxas de prisões do DFV ocorreram onde as chefes de polícia estavam presentes em uma força policial (Andrews e Miller 2013, 1010). É importante ressaltar que "a qualidade de vida das mulheres, como vítimas de violência doméstica, pode ser muito melhorada se houver maior representação das mulheres dentro do serviço policial" (Andrews e Miller 2013, 1011). Os autores descobriram que as mulheres policiais que tinham maior autoridade e oportunidade de exercer discrição representavam ativamente os interesses das mulheres (Andrews e Miller 2013, 1010). Pode ser que o enfrentamento do equilíbrio de gênero dentro do serviço policial possa auxiliar no incentivo às mudanças culturais e melhores respostas ao DFV. O Serviço de Polícia de Queensland é uma instituição dominada por homens. A revisão estatística mais recente realizada pelo Serviço de Polícia de Queensland (2017a) mostra que, embora quase metade dos novos recrutas sejam mulheres, há um evidente desequilíbrio de gênero em níveis mais altos do serviço. Aproximadamente $30 \%$ dos policiais são mulheres, $21 \%$ dos oficiais não comissionados são mulheres e há apenas uma mulher (seis por cento) do Executivo da Polícia de Queensland de 18 membros (Serviço de Polícia de Queensland 2017a).2Mais, dos 44 membros de vários comitês do QPS, seis (13 por cento) são mulheres, sendo três dessas mulheres membros externos que não são funcionários do QPS (Serviço de Polícia de Queensland 2017b). Com o movimento para um serviço policial mais equilibrado de gênero no final do recrutamento, podemos observar algumas mudanças na forma como o policiamento é conduzido em Queensland. No entanto, garantir que as mulheres tenham maior autoridade dentro do serviço policial pode ser uma maneira mais confiável de melhorar o policiamento do DFV.

Vários estudos (Barberet e Carrington 2018; Perova e Reynolds 2015 destacaram o trabalho das centenas de delegacias de delegacias de mulheres (WPSs) em toda a América Latina. Os WPSs têm sido desenvolvidos desde a década de 1980 para "aumentar a confiança das mulheres no sistema de justiça criminal, incentivar a notificação, prevenir a revitimização e enviar uma mensagem à comunidade de que a violência de gênero não era mais tolerada e os homens que abusam das mulheres serão responsabilizados" (Barberet e Carrington2018, 837). Os WPSs operam usando uma variedade de modelos, mas suas características comuns incluem que as estações geralmente estão localizadas separadamente das delegacias regulares, as operadoras recebem treinamento especializado e os operadores são capazes de coordenar o acesso das mulheres a uma gama de serviços, incluindo serviços que prestam apoio através do sistema de justiça, aconselhamento, treinamento de habilidades de trabalho e cuidados com crianças (ONU Mulheres 2011, 2). A ONU Mulheres $(2011,4)$ relata que os WPSs melhoraram o acesso das mulheres à justiça, resultando em mais acusações contra abusadores violentos, e a maioria dos usuários do WPS entrevistados relatou sentir que foram ouvidos e, como resultado, melhor apoiados e compreendidos. A maioria dos usuários também percebeu que os WPSs contribuíram para a redução da violência contra as mulheres (ONU Mulheres 2011, 4). Perova e Reynolds (2015) examinaram dados de mortalidade nacional no Brasil para determinar o efeito das PMSs no homicídio de mulheres. Eles concluíram que os WPSs tiveram fortes efeitos nas taxas de homicídios entre mulheres de 15 a 24 anos, residentes em áreas metropolitanas maiores, resultando em uma redução de $70 \%$ na taxa média de homicídios para mulheres desse grupo (Perova e Reynolds 2015, 17). 
O Relatório Not Now, Not Ever(2015, 50) reconheceu que 'fatores culturais e atitudinais ... [afetar]o comportamento dos profissionais dentro de nossos sistemas de polícia, justiça, bem-estar e prestador de serviços quando chamados a responder e gerenciar a violência doméstica. A polícia é apenas uma das respostas ao DFV, mas são importantes. Esta pesquisa sugere que ainda há um caminho a percorrer para efetivar a mudança cultural necessária entre as polícias. No contexto de Queensland, foram introduzidos treinamentos e unidades especializadas da DFV e há um número crescente de policiais no terreno. No entanto, os relatos de mortes associadas ao DFVand permanecem persistentemente altos (Governo de Queensland 2017, 35). Talvez seja hora de reconsiderar a mensagem de recrutamento da polícia e a representação de mulheres em altos escalões do serviço policial. Também pode ser hora de investigar o potencial das únicas delegacias femininas. 